\title{
Zhao Tingyangs Alles unter dem Himmel: Eine kritische Auseinandersetzung ${ }^{1}$
}

\section{Zhao Tingyang's All Under Heaven: A Critical Examination}

\section{Philippe Brunozzi, Nanjing / Kassel \& Henning Hahn, Berlin}

Zusammenfassung: Zhao Tingyang gehört zu den einflussreichsten Denkern der chinesischen Gegenwartsphilosophie. In seinem 2020 bei Suhrkamp erschienenen Werk (Alles unter dem Himmel: Vergangenheit und Zukunft der Weltordnung) reaktualisiert er die klassische Idee des Tianxia („Alles unter dem Himmel“). Zhao schlägt darin einen Perspektivenwechsel vor, nach dem die globale Ordnung jenseits der Idee von Nationalstaaten als radikal integrierte Ganzheit zu betrachten ist. Die folgende Buchdiskussion begibt sich in die Auseinandersetzung mit Zhaos interessantem, aber auch provokantem Vorschlag. Einleitend werden Philippe Brunozzi und Henning Hahn zunächst Zhaos Grundgedanken herausarbeiten. Er konzipiert die Welt insgesamt als politisches Subjekt, stellt dem westlichen Rationalitätsbegriff eine relationale Rationalität gegenüber und entwirft mit dem Tianxia-System ein globales Ordnungsmodell. Es schließen sich kritische Kommentare von Stefan Gosepath, Walter Pfannkuche, Luise Müller, Christian Neuhäuser, Hans Feger und Robin Celikates an, auf die Zhao Tingyang abschließend antwortet.

1 Die hier dokumentierte Buchdiskussion resultiert aus einer im November 2019 an der FU Berlin abgehaltenen Tagung mit Zhao Tingyang. Wir danken Hans Feger und Stefan Gosepath für ihre tatkräftige Unterstützung. Die Tagung fand im Rahmen des vom BMBF geförderten und von Hans Feger und Stefan Gosepath geleiteten Alumni-Netzwerks für Geistes- und Sozialwissenschaftler - das gute Leben - statt. Auch danken wir für die Unterstützung der von der DFG geförderten Kolleg-Forschergruppe Justitia Amplificata. 
Schlagwörter: Zhao Tingyang, Tianxia („Alles unter dem Himmel“), relationale Rationalität, konfuzianisches Optimum, Minxin („Volksseele“), globale Philosophie, chinesische Philosophie, Kosmopolitismus

Abstract: Zhao Tingyang is one of the most influential thinkers of contemporary Chinese philosophy. In his work, he revisits the classical idea of Tianxia ("all under heaven"). In it, Zhao proposes a change of perspective, according to which the world is to be seen as a radically integrated whole. The following book discussion takes up Zhao's thought-provoking proposal. Philippe Brunozzi and Henning Hahn will begin by elaborating the book's basic ideas. Zhao conceives of the world itself as a political subject, contrasts the Western conception of rationality with the notion of a relational rationality and designs a global order beyond. This is followed by critical commentaries by Stefan Gosepath, Walter Pfannkuche, Luise Müller, Christian Neuhäuser, Hans Feger and Robin Celikates, to which Zhao Tingyang finally responds.

Keywords: Zhao Tingyang, tianxia ("all under heaven"), relational rationality, Confucian optimum, global philosophy, Chinese philosophy, cosmopolitanism

Zhao Tingyang gehört zu den einflussreichsten und originellsten Denkern der chinesischen Gegenwartsphilosophie. Der Professor für Philosophie an der Chinesischen Akademie der Sozialwissenschaften in Peking widmet sich in seinem umfangreichen Werk klassischen Themen der politischen Philosophie, wie politischer Legitimität, Menschenrechten, der Möglichkeit stabiler Kooperation angesichts konkurrierender Wertesysteme und der Begründung universeller Werte. Kennzeichnend für sein Werk ist, dass er eine politische Wende der Philosophie vertritt und in dieser modernen Zielsetzung immer wieder auf Grundkonzepte aus der chinesischen philosophischen Tradition zurückgreift. So auch in seinem 2016 erschienenen Werk 天下的当代性:世界 秩序的实践与想象 (Alles unter dem Himmel: Vergangenheit und Zukunft der Weltordnung), das mit Rückgriff auf die klassische Vorstellung des „Tianxia“ („Alles unter dem Himmel“) das Modell einer alternativen Weltordnung entwirft. Zhao schlägt darin einen Perspektivenwechsel vor, nach dem die globale Ordnung jenseits der Idee von Nationalstaaten als radikal integrierte Ganzheit zu betrachten ist.

Zhao zählt zu den wenigen chinesischen Gegenwartsdenkern, die in von angloeuropäischen Autor*innen dominierten Debatten breiteres Gehör finden. Das zeigt sich nun auch an der sorgfältig von Michael Kahn-Ackermann übersetzten Herausgabe von Alles unter dem Himmel: Vergangenheit und Zukunft der Weltordnung in der STW-Reihe des Suhrkamp Verlags 
(2020). Die Veröffentlichung ist in vielerlei Hinsicht diskussionswürdig. Auf den ersten Blick deshalb, weil sie einer scheinbar politisch problematischen Position ein Forum bietet. In seinem Feature im Deutschlandfunk weist zum Beispiel Tobias Wenzel darauf hin, dass Zhaos politische Philosophie im Einklang mit dem selbstbewusster werdenden außenpolitischen Anspruch Chinas steht. ${ }^{2}$ Unabhängig davon, ob diese Einschätzung zutrifft, muss von Anfang an klar sein, dass die philosophische Reaktualisierung des Tianxia-Systems im Spannungsfeld global sich verschiebender Machtverhältnisse zu lesen ist. Ihre Diskussion gibt zunächst einmal Aufschluss über ein sich wandelndes politisches Selbstverständnis.

Wir halten die Schrift aber auch in philosophischer Hinsicht für diskussionswürdig. Erstens und generell ist die Veröffentlichung Ausdruck einer sich verändernden Diskurslandschaft. Es ist an der Zeit, dass die Orientierung der politischen Philosophie an angloamerikanischen Autor*innen diversifiziert und von einem globalen Diskurs flankiert wird. China wird darin eine bedeutende Rolle spielen und es lohnt sich, einen originären und einflussreichen chinesischen Denker zu erkunden. Zweitens und in Hinsicht auf den philosophischen Ertrag ist es unseres Erachtens durchaus instruktiv, sich mit Zhaos spezifischer Konzeption des Tianxia auseinanderzusetzen. Handelt es sich doch um eine kosmopolitische Philosophie, die gezielt ,westliche ${ }^{6}$ er nutzt dieses Prädikat allerdings oftmals recht einseitig - Ordnungsmodelle herausfordert.

So weist beispielsweise Bernhard Zand in seiner Spiegel-Rezension (08/2020) darauf hin, dass hinter den Kulissen einer in Passagen zwar unscharf bleibenden und oftmals sicherlich pauschalisierenden Schrift die Kontur einer alternativen Denkweise und mit ihr einer alternativen Weltordnung erkennbar wird. Die Auseinandersetzung mit dieser Alternative erscheint uns ebenso notwendig wie fruchtbar zu sein. Einleitend wollen wir daher einem hermeneutischen principle of charity folgen: Worin liegt die ernstzunehmende Herausforderung von Zhaos Denken?

Dazu werden wir zunächst (a) grundsätzliche Überlegungen zur globalen politischen Philosophie voranstellen, um kurz (b) in die philosophischen und (c) politischen Grundgedanken von Zhaos Tianxia-Konzeption einzuführen. Es folgen kritische Kommentare von Stefan Gosepath, Walter

2 https://www.deutschlandfunkkultur.de/zhao-tingyang-alles-unter-dem-himmel-weltfrieden-auf.1270.de.html?dram:article_id=468415 (Zugriff 03.2020). 
Pfannkuche, Luise Müller, Christian Neuhäuser, Hans Feger und Robin Celikates, zu denen Zhao Tingyang abschließend Stellung bezieht.

a) Globale politische Philosophie: Die Auseinandersetzung mit Zhao Tingyangs politischer Philosophie ist in eine der gegenwärtig wichtigsten Theorieentwicklungen eingebettet: dem global turn in der politischen Philosophie. In ihrem Artikel If Philosophy Won't Diversify, Let's Call It What It Really Is (2016) monieren Jay Garfield und Bryan Van Norden, dass die gegenwärtige Philosophie, jedenfalls wie sie in den meisten angloeuropäischen Instituten gelehrt wird, ein ernstes Diversitätsproblem aufweist. Dass philosophische Traditionen Afrikas oder Asiens im angloeuropäischen Diskurs unterrepräsentiert sind, wirkt nicht nur aufgrund einer zunehmend multipolaren Welt, sondern schon angesichts der Diversität in unseren Seminaren anachronistisch. Der Fokus auf einen ,westlichen“ Kanon ist aus dreierlei Gründen zu überwinden: Erstens stellt er eine epistemische Ungerechtigkeit dar, in dem Sinne, dass er sprachliche, wissenschaftspolitische und methodische Ausschlüsse vornimmt, die globale Ungerechtigkeiten fortschreiben. Zweitens ist das nicht nur unfair, sondern auch sachlich nicht zu rechtfertigen. ${ }^{3}$ Die Ignoranz gegenüber chinesischen und anderen philosophischen Traditionen verspielt ein Reservoir von Erkenntnissen, Erfahrungen und Herangehensweisen, das mindestens als Irritationspunkt genutzt werden sollte. Drittens und entscheidend ist aber, dass globale Philosophie nicht gleichbedeutend mit komparativer Philosophie ist, sondern systematisch auf ihr aufbaut. Es geht nicht nur um interkulturelle Kompetenz, also um das Verstehen des anderen als anderen, sondern um ein gemeinsames Philosophieren als einer unter Gleichen und vor dem Hintergrund einer verbundenen Lebenswelt. In diesem Sinne bedeutet globale Philosophie, dass wir alle zur Verfügung stehenden philosophischen Ressourcen nutzen sollten, um geteilte theoretische und praktische Probleme zu lösen.

In Philosophy Unbound: The Idea of Global Philosophy fasst Thom Brooks dieses Projekt wie folgt zusammen: „The future of philosophy is moving towards ,global philosophy'. The idea of global philosophy is the view that different philosophical approaches may engage more substantially with each other to solve philosophical problems“ (Brooks 2013, 254). Es geht darum, sich auf einen Diskurs einzulassen, in dem sich unterschiedliche Traditionen wechselseitig herausfordern und befruchten - und zwar nicht, um einen

3 Gegen das Branding als Welt- oder Ethnophilosophie polemisiert beispielsweise Hamid Dabashi 2015. 
Ansatz als den überlegenen hervortreten zu lassen, und auch nicht nur, um die Eigenart des anderen zu verstehen, sondern um eine gemeinsame Diskurspraxis zu etablieren, in der das Zusammenspiel unterschiedlicher Traditionen neue Sichtweisen ermöglicht. In diesem Sinne unterscheidet auch Tim Connolly (2015) komparative von globaler Philosophie und drückt ihre Differenz in Musikgenres aus: Während komparative Philosophie Welt- und Ethnomusik archiviert und für unsere Ohren aufbereitet, mischt globale Philosophie Samples aus unterschiedlichen Traditionen zu neuen Klängen. Dabei kann globale Philosophie unterschiedliche Ausprägungen annehmen, je nach philosophischer Orientierung und Absicht. Als globale politische Philosophie trägt sie damit nicht zuletzt zur Herausbildung einer globalen Öffentlichkeit bei, in der sich interkulturelle Identitäten entwickeln und gemeinsame politische Erfahrungen artikuliert und problematisiert werden können (siehe Williams und Warren 2014). Dass die Praxis der globalen Philosophie nicht reibungslos verläuft, ist offensichtlich. Immer wieder muss sie aus dem eigenen Diskursrahmen heraustreten, um koloniale Vorstrukturierungen, subtile Machtasymmetrien und verzerrende Vorannahmen aufzuspüren.

Im Grunde hat sich Zhao Tingyang mit seiner Schrift bereits dieser Idee einer globalen Philosophie verschrieben. Er nimmt, westliche“ Theorien auf und übersetzt die Tradition des Tianxia - oftmals mit Hilfe innovativer Neologismen - in ein modernes Problemverständnis. Der Punkt einer Auseinandersetzung mit Zhao ist unseres Erachtens nicht, ob seine oder eine der von ihm angegriffenen ,westlichen“ Konzeptionen recht hat, sondern ob sich aus dem damit eröffneten Diskurs zwischen den Traditionen ein besseres, weil achtsames Verständnis über eines der drängendsten Zukunftsprobleme ausbilden kann: die Frage nach der Einrichtung und Legitimität robuster globaler Governance-Prozesse.

b) Die Philosophie des Tianxia: „Alles unter dem Himmel“ (tianxia 天下) ist ein Begriff der chinesischen Antike. Er entstand, so Zhao, vor etwa 3000 Jahren unter der Zhou-Dynastie und bezeichnet eine Herrschaftsform, das Tianxia-System, in der nicht ein Volk alle anderen unterjocht, sondern eine gemeinsame Welt für alle schafft. ${ }^{4}$ Bevor wir uns aber den politischen Implikationen des Tianxia-Systems zuwenden, möchten wir zwei ihrer philosophischen Grundgedanken voranstellen. Denn Zhaos Reaktualisierung des Tianxia ist für ihn weniger ein politisches, denn ein ideographisches Pro- 
jekt; im Vordergrund steht eine neue begriffliche Ausmessung politischer Subjektivität und Rationalität.

Der erste, schwer zu greifende Grundgedanke ist der, dass die Welt selbst zum politischen Subjekt werden soll. Tianxia als „Welt der Weltheit“ (a world of worldliness) bezeichnet eine Perspektivenverschiebung in der Weltpolitik, die nicht im Interesse einzelner Staaten, sondern aus dem Interesse der Welt selbst betrieben wird: „Das Konzept des Tianxia zielt auf eine Weltordnung, worin die Welt als Ganzes zum Subjekt der Politik wird, auf eine Ordnung der Koexistenz (order of coexistence), welche die ganze Welt als eine politische Entität betrachtet" (Zhao 2020, 14). Hier wird eine holistisch-inklusive Perspektive vorausgesetzt, die nicht nur klassisch chinesische Vorstellungen transportiert, sondern an unterschiedliche kosmopolitische Denkmuster angeschlossen werden kann. Kosmopolitismus, ernst genommen, setzt eine Harmonisierung der Interessen und eine weltbürgerliche Identifikation voraus, ein Denken als Welt, das Zhao im Tianxia-System präfiguriert findet.

Der zweite hervorzuhebende Grundgedanke ist der einer relationalen Rationalität. Diese wird dem egozentrischen Verständnis von Rationalität entgegengestellt, wie es nach Zhao die politische Philosophie des Westens und insbesondere den Kontraktualismus kennzeichnet. Statt einer individuellen Nutzenmaximierung und insgesamt einer Orientierung am Individuum stehen im relationalen Rationalitätsverständnis die Einsicht in den Vorrang einer ursprünglichen Koexistenz mit anderen und das Interesse an einem friedlichen Zusammensein im Vordergrund. Die Ausbildung eines „koexistentiellen Bewusstseins“ (Zhao 2020, 41) sei, so Zhaos spieltheoretisches Argument, die überlegene Strategie, weil sie zukünftiges Vertrauen und wechselseitigen Nutzen maximiert. Diesen Zustand bezeichnet er als konfuzianisches Optimum: „Relationale Rationalität (...) dient der Schaffung stabiler und auf Vertrauen gegründeter Koexistenz, ihr ideales Ziel ist das ,konfuzianische Optimum ' (Confucian Improvement). Es besagt, dass an einer Nutzenverbesserung stets alle an der Angelegenheit beteiligten Personen partizipieren müssen“ (ebd.). Allgemeines Einverständnis (Legitimität) ergibt sich daraus, dass eine Entscheidung der ,Volksseele (minxin 民心) entspricht. Wie einige der folgenden Kommentare kritisch anmerken, ist dieser Gedanke harmonistisch und demokratiekritisch zu verstehen. Darin scheint Zhaos Ansatz aber kommunitaristischen und insbesondere Hegelianisch inspirierten Kritiken des Liberalismus durchaus nahezustehen. Ganz gleich also, ob der Liberalismus verteidigt, überwunden oder weiterentwickelt wer- 
den soll, lässt sich die philosophische Auseinandersetzung mit Zhaos holistischer und relationaler Denkweise nicht umgehen.

c) Die Politik des Tianxia: Die politische Beurteilung von Zhaos Tianxia-System folgt drei Hinsichten, nämlich seiner historischen, normativen und realpolitischen Bedeutung. Historisch ähnelt es der Pax Romana, insofern es ein stammesübergreifendes Herrschaftssystem errichtet, in dem ein Kronland ein Netz von Vasallenstaaten politisch beherrscht und rechtlich integriert. Normativ handelt es sich um die realistische Utopie einer kosmopolitischen Ordnung. Auf die Frage, wie globale Herrschaft zugleich möglich, robust und legitim sein kann, antwortet das Tianxia-System mit der Idee eines dynamischen Netzwerks, dem sich Staaten zum eigenen Nutzen anschließen. Der Führungsanspruch kann jederzeit wechseln und beruht nicht (allein) auf Macht, sondern auf Systemüberlegenheit und Inklusion in ein System wechselseitigen Nutzens:

1. Das Tianxia-System musste sicherstellen, dass der Beitritt zum System für jeden Staat mehr Vorteile schaffte als das Draußenbleiben, es daher von allen Staaten akzeptiert wird und der Beitritt freiwillig erfolgte.

2. Ein Tianxia-System musste in der Lage sein, Beziehungen wechselseitiger Abhängigkeit und gegenseitiger Begünstigung hinsichtlich der Interessen zu schaffen, um dadurch eine Ordnung allgemeiner Sicherheit und dauerhaften Friedens der Welt zu gewährleisten.

3. Ein Tianxia-System musste in der Lage sein, umfassend günstige Voraussetzungen für das Gemeinwohl, die Nutzenteilhabe und die öffentlichen Angelegenheiten aller Staaten zu entwickeln, um so den universellen Teilhabecharakter des Tianxia-Systems unter Beweis zu stellen (Zhao 2020, 56f.).

Was die Umsetzung dieses Ordnungsmodells durch konkrete Institutionalisierungsschritte betrifft, bleibt Zhaos Entwurf eher blass. In früheren Schriften deutet er an, dass ein heutiges Tianxia-System aus einer Weltregierung besteht, die Aufgaben der Weltinnenpolitik übernimmt, in die Souveränität einzelner Staaten (substates) aber nur dann eingreift, wenn sie universelle Gesetze brechen (siehe Zhao 2009).

Zuletzt ist es interessant, dass Zhao in Alles unter dem Himmel einen realistischen Weg zum Tianxia-System andeutet, da er die Menschheit an der Schwelle einer globalen technologischen Diktatur sieht, die eine Institutionalisierung des Tianxia-Systems immer nötiger werden lässt. Während sich diese Passagen auch als Kritik an China lesen lassen, argwöh- 
nen die folgenden Kommentare, dass Zhao einer Pax Sinica Tür und Tor öffnet. Bezeichnend ist, dass Tianxia in den letzten Jahren zum Leitbegriff außenpolitischer Thinktanks in China avanciert ist (siehe Zhang 2015; Wang 2013; Babones 2017). Und in der Tat lässt sich der neue Führungsanspruch Chinas, der Stabilität und Wohlstand statt Demokratie und Freiheit verspricht, anhand Zhaos Überlegungen besser einordnen. Klar ist, dass sich Zhao gegen solche Instrumentalisierungsversuche ausdrücklich verwahrt hat. Weniger klar ist, welche Sicherungen seine Theorie bereithält, um hegemoniale Vereinnahmungen abzuwehren. Aber selbst wenn - oder gerade weil - uns Alles unter dem Himmel letztlich auf eine postliberale Weltordnung vorbereiten will, lohnt es sich umso mehr, sich damit zu befassen.

\section{Literatur}

Babones, Salvatore. 2017. American Tianxia: Chinese Money, American Power, and the End of History. Bristol: Policy Press.

Brooks, Thom. 2013. „Philosophy Unbound: The Idea of Global Philosophy“. Metaphilosophy 44, 254-266.

Connolly, Tim. 2015. Doing Philosophy Comparatively. London: Bloomsbury.

Dabashi, Hamid. 2015. Can Non-Europeans Think?, London: Zed Books.

Garfield, Jay L., und Bryan W. Van Norden. 2016. „If Philosophy Won't Diversify, Let's Call It What It Really Is“. The Stone, The New York Times, 11. Mai 2016.

Wang, Ban. 2017. Chinese Visions of World Order. Tianxia, Culture, and World Politics. Durham: Duke University Press.

Wang, Gungwu. 2013. Renewal. The Chinese State and New Global History. Hongkong: The Chinese University of Hong Kong Press.

Williams, Melissa, und Mark Warren. 2014. „A Democratic Case for Comparative Political Theory“. Political Theory 42, 26-57.

Zhang, Feng. 2015. Chinese Hegemony: Grand Strategy and International Institutions in South East Asia. Stanford: Stanford University Press.

Zhao, Tingyang. 2009. „A Political World Philosophy in terms of All-under-heaven (Tian-xia)“. Diogenes 56, 5-18.

---. 2020. Alles unter dem Himmel: Vergangenheit und Zukunft der Weltordnung, Berlin: Suhrkamp. 